\title{
Application SimlnTech Software for Optimization Fuel System Parameters of the Perspective Helicopter
}

\author{
Anatoly Satin ${ }^{1}$, Roman Savelev ${ }^{1 *}$, Denis Smagin ${ }^{1}$, Konstantin Napreenko ${ }^{1}$ and Anastasia \\ Neveshkina ${ }^{1}$ \\ ${ }^{1}$ Moscow Aviation Institute (National Research University), Moscow, Russia
}

\begin{abstract}
The possibility of applying optimization algorithms for mathematical modelling of the helicopter fuel system on the example of the advanced helicopter in Russian software complex SimInTech. The results of the fuel system parameters simplest optimization for the nominal operation mode are presented, examples of algorithms for the selection of the parameters of the units are shown.
\end{abstract}

\section{Introduction}

The creation of modern types of aviation technology is a complex and multi-iterative process, in which successful development and further product operation can be ensured only if longterm and thorough design studies and experimental tests are carried out.

Currently, mathematical modeling is a rapidly developing method of studying the behavior of complex systems.

Using mathematical modeling for the design of aircraft's on-Board systems allows:

- $\quad$ reduce time for system design;

- optimize the system architecture according to the criteria of weight and energy perfection;

- formulate requirements to the suppliers of components;

- develop and optimize control algorithms;

- $\quad$ assess the reliability and fault safety of on-board systems.

Mathematical modeling is a cyclic and iterative process, i.e. the mathematical model of the object or system is expanded and refined with the appearance or refinement of the initial data, and the initial mathematical model is gradually improved, complicated and approaching the real system.

Iteratively process of mathematical modeling is well illustrated by the design process: typically, firstly a system is designing and then testing, after re-adjust the project with test results until the project will not meet its requirements.

Fuel system of the advanced helicopter is designed to:

- placing the fuel in the fuel tanks;

- fuel tank ventilation;

- fuel delivery to the engine;

- centralized or separate filling of fuel tanks;

*Corresponding author: mailto: r_sr@inbox.ru, maksmai33@gmail.com 
- $\quad$ centralized or separate draining fuel from the tank;

- Management and control.

Special software is used to solve the problems of structural and functional parameters optimization of fuel systems. Such tool is allowing to replace real complex systems and structures with structural schemes in the form of blocks of mathematical models that fully describe these systems and structures and allow to vary the parameters of the system to obtain the optimal value in accordance with the requirements. $[1,2]$

As optimization of parameters in this article selection is carried out:

- $\quad$ the diameters of the refuel subsystem pipelines

- the fuel pump pressure to achieve a predetermined difference between the fuel consumption entering the fuel consumed tank and taken from the fuel consumed tank (maintaining a predetermined level in the fuel consumed tank tank)

Adjustment of parameters is carried out using a simple algorithm that works on the signal mismatch between the required and the current fuel consumption. Optimization in this case means selection of geometrical parameters of pipelines and parameters of fuel pump with minimum resistance. [3]

\section{Methodology of research}

The SimInTech software refers to computer-aided design (CAD) systems of logic-dynamic systems described in input-output relations and has enough functionality to create models of complex technical systems with a specified accuracy.

The problem of optimization is determined, first, by the difficulty of its formalization. A formal approach (for example, by folding several criteria into one) often leads to an unacceptable solution. Therefore, the parametric optimization procedure should be organized in such a way that the Designer can intervene at any time and adjust the specified technical requirements. At the same time, this procedure should be based on the use of known methods of parametric optimization.

Proceeding from the aforesaid, it is necessary to organize the decision of a problem of parametrical optimization in the form of dialogue iterative procedure. The parametric optimization dialogue procedure can be reduced to two operations in turn:

- adjustment of the terms of reference for optimal planning;

- solution of the parametric optimization task according to the specified technical requirements;

- denote $x_{1}, x_{2}, \ldots, x_{n}$ - parameters of the system under design, which can be changed during optimization to ensure the specified quality. Each parameter can be limited by the view

$$
x_{i, \min } \leq x i \leq x_{i, \max }
$$

Let's just as well $Q_{1}(x), Q_{2}(x), \ldots, Q_{m}(x)$ - quality indicators (criteria), calculated as a result of modeling at given values of parameters.

The requirement to each of the criteria will be set in the form of restrictions

$$
Q_{i, \min } \leq Q_{i}(x) \leq Q_{i, \max }
$$

Let's formulate the task of parametric optimization as follows: to find the vector of parameters $x^{*}=\left[x_{1}^{*}, \ldots, x_{n}^{*}\right] T$, satisfying the limitations (1.1), where the quality indicators meet the limitations (1.2). Adjustment may consist in changing the set of optimized parameters and criteria, as well as in changing the boundary values in the restrictions (1.1), (1.2).

The efficiency of optimization is determined, first, by the conditionality of the task. The conditionality of the task also depends heavily on the selected scale of parameters and criteria. Indeed, the parameters and criteria may be of very different physical nature, and the 
range of their changes may vary significantly. Therefore, only a change of scale and transition to normalized values often leads to a significant increase in the efficiency of optimization.

Setting the ranges of parameter changes and criteria considering their physical meaning allows for their natural scaling. For example, the initial steps for the parameters are set as follows:

$$
\Delta x_{i}=0,1\left(x_{i, \max }-x_{i, \min }\right)
$$

Effective algorithms usually carry out the reorganization of the coordinate system in the process of optimization, however, a successful choice of scale significantly increases the efficiency at the initial stages of search.

Similarly, the quality criteria should be scaled up. We form normalized private criteria in the form:

$$
q_{i}(x)=\left\{\begin{array}{l}
0, Q_{i, \min } \leq Q_{i}(x) \leq Q_{i, \max }, \\
\frac{Q_{i}(x)-Q_{i, \max }}{Q_{i, \max }-Q_{i, \min }}, Q_{i}(x)>Q_{i, \max }, \\
\frac{Q_{i, \min }-Q_{i}(x)}{Q_{i, \max }-Q_{i, \min }}, Q_{i}(x)<Q_{i, \min } .
\end{array}\right.
$$

In this case, the normalized criteria (1.4) accept zero values if the corresponding limits (1.2) are fulfilled and the positive values otherwise.

To solve a multicriteria optimization task, we usually pass from several specific criteria $q_{1}, \ldots, q_{m}$ to one common criterion, which is formed as a function of partial criteria. This procedure is called a curtailment of criteria. As a result, we get a general criterion (target function)

$$
f(x)=j\left(q_{1}(x), \ldots, q_{m}(x)\right)
$$

as a function of the optimized parameters. The solution of the multicriteria optimization task is to minimize this criterion. There are many ways to curtail private criteria. Here is one of them, summarizing many known ways. The general criterion can be formed in the form of an average degree of optimality criterion.

$$
\varphi_{\mathrm{p}}\left(\mathrm{q}_{1}, \ldots, \mathrm{q}_{\mathrm{m}}\right)=\left[\frac{1}{\mathrm{~m}} \sum_{\mathrm{i}=1}^{\mathrm{m}} \mathrm{q}_{\mathrm{i}}^{\mathrm{p}}\right]^{1 / \mathrm{p}} .
$$

If $\mathrm{p}=1$, we obtain the additive criterion

$$
\varphi_{1}\left(\mathrm{q}_{1}, \ldots, \mathrm{q}_{\mathrm{m}}\right)=\frac{1}{\mathrm{~m}} \sum_{\mathrm{i}=1}^{\mathrm{m}} \mathrm{q}_{\mathrm{i}} .
$$

At $\mathrm{p}=2$, we obtain a quadratic criterion

$$
\varphi_{2}\left(\mathrm{q}_{1}, \ldots, \mathrm{q}_{\mathrm{m}}\right)=\left[\frac{1}{\mathrm{~m}} \sum_{\mathrm{i}=1}^{\mathrm{m}} \mathrm{q}_{\mathrm{i}}^{2}\right]^{1 / 2}
$$

With $\mathrm{p}$ striving for infinity, the general criterion (1.6) is reduced to the largest of the normalized partial criteria (minimax criterion)

$$
\varphi \infty\left(q_{1}, \ldots, q_{m}\right)=\max \left(q_{1}, \ldots, q_{m}\right) .
$$

At $\mathrm{p}=0$, logarithmizing the expression (1.6) and moving to the $\mathrm{p}$ limit, which tends to zero, after applying the Lopital rule we obtain the mean geometric (multiplicative) optimality criterion 


$$
\varphi_{0}\left(q_{1}, \ldots, q_{m}\right)=\left[\prod_{i=1}^{m} q_{i}\right]^{1 / m} .
$$

It can be shown that for the average power criterion (1.6) at any $\mathrm{p}$ the optimal solution $\mathrm{x}^{*}$ of the parametric optimization problem

$$
\varphi_{p}\left(q_{1}(x), \ldots, q_{m}(x)\right) \rightarrow \min (1.11)
$$

is an effective point and belongs to many Paretoes of the initial task of multi-criteria optimization.

Optimization methods depending on the order of derivatives used are divided into zero, first and second order methods.

Zero-order methods (direct methods or search methods) use only function values. Among the most effective methods of direct search - the methods of Rosenbrock and Powell, configurations, method of Hooke-Jeeves, simplex method of Nelder-Mead, the complex method of Box. All of them are heuristic and are particularly effective in cases where other methods with high convergence rate are unable to solve the problem, for example, if the minimized function is not smooth.

Methods of the first order (gradient) use, in addition to the values of the function, the values of its derivatives. The most effective among gradient methods are variable metric or quasi-Newtonian methods based on the approximation of the second derivative matrix or the inverse of it. Gradient methods are useful in cases where it is possible to calculate the derivatives of the minimized function.

The second-order (Newtonian) methods additionally require the computation of second derivatives, have quadratic convergence, and converge rapidly if the function is convex and sufficiently smooth. However, these methods may be ineffective or even divergent if the function is not convex. The second order methods should be used only in cases when it is easy enough to obtain the matrix of the second derivatives of the minimized function.

The use of first and second order methods requires the calculation of derivatives of the General quality criterion. If the criterion is computed according to the simulation results, it is possible to calculate derivatives or numerically, giving the increment parameters, or by equations of sensitivity. Numerical calculation of derivatives requires a large amount of computation and often leads to significant errors. The use of sensitivity equations causes an increase in the order of the system under study and requires additional memory. Certain difficulties are encountered in modeling the sensitivity of systems containing ambiguous and discontinuous characteristics (backlash, dry friction, relay). Therefore, it is advisable to use zero-order methods for optimization based on simulation results. [4,5,6]

\section{A mathematical model of the advanced helicopter fuel system. Optimization of fuel system parameters.}

A dynamic model of the advanced helicopter fuel system was developed in SimInTech software package. This model includes a thermal hydraulic circuit (contains fuel tanks, fuel pumps, jet pumps, pipelines, etc.), a control circuit (algorithms for opening/closing dampers, on/off electric pumps, etc.) and a database of signals (a list of all signals for algorithms and data exchange between the automation circuit and the thermal hydraulic circuit) $[3,4]$. Fuel tanks are divided into two groups (right, left) consisting of three separate tanks.

Figure 1 shows the scheme of the helicopter fuel system thermal hydraulic. As a first example for the optimization of hydraulic schemes (it can be any scheme: air conditioning system, hydraulic system, neutral gas system, etc.) is considered the selection of refueling pipelines diameters (Figures in purple show the threading pipes with varying flow rates; the numbers correspond to the sensors in Figures 2-3). It is considered the option of such a filling in which completely different costs are -required in each pipeline to demonstrate the 
operation of the selection algorithm - we will set different refueling costs for each of the 6 tanks (see table 1). [7,8]

Figure 2 shows the simplest algorithms for selecting pipe diameters. Based on the mismatch signal between the specified fuel consumption value and the current value, the process of determining each pipeline diameter is started. The optimization method described in chapter 2 enables diameters to be matched to a given flow rate in the shortest possible time, thus forming a given fuel consumption distribution.

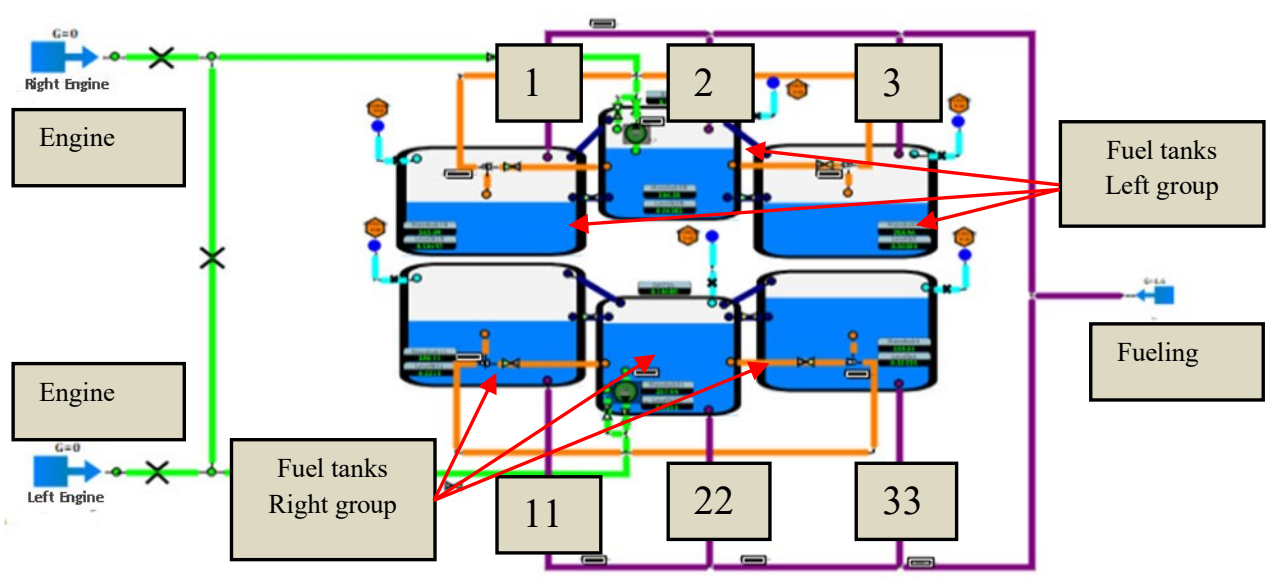

Fig. 1. The scheme of the helicopter fuel system thermal hydraulic

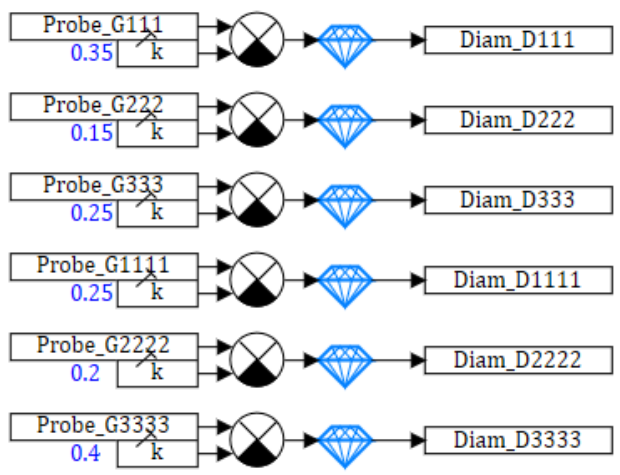

Fig. 2. The simplest algorithms for selecting pipe diameters

Figure 3 shows a graph of the fuel consumption change in each pipeline depending on the model counting time; figure 4 shows the values of the pipeline diameters for achieving the specified costs. Table 1 shows that the specified calculations are achieved accurately. Thus, it is possible to determine the geometric parameters of the systems for the distribution of the coolant (fuel, air, oil) in different systems. 


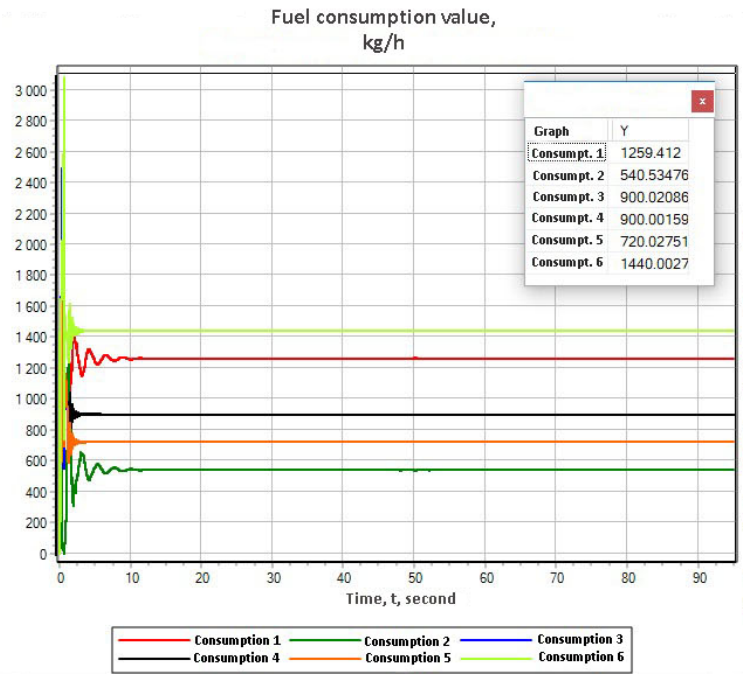

Fig. 3. A graph of the fuel consumption changes in each pipeline depending on the model counting time

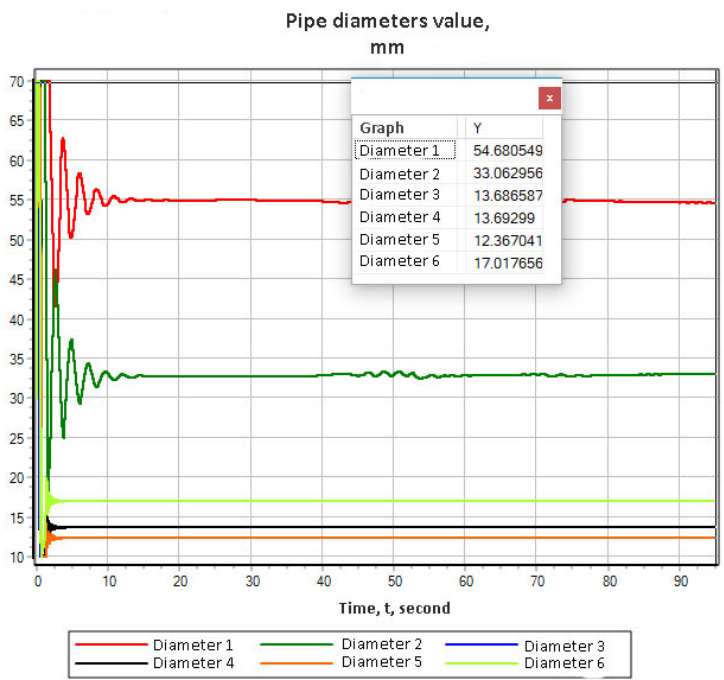

Fig. 4. The values of the pipeline diameters

Table 1. The specified calculations

\begin{tabular}{|c|c|c|c|}
\hline Parameter & $\begin{array}{c}\text { Fuel tank } \\
\text { (Figure 1) }\end{array}$ & $\begin{array}{c}\text { Specified } \\
\text { consumption value, } \\
\mathrm{kg} / \mathrm{h}\end{array}$ & $\begin{array}{c}\text { Calculated } \\
\text { consumption value, } \\
\mathrm{kg} / \mathrm{h}\end{array}$ \\
\hline Consumption 1 & 1 & 1260,0 & 1259,4 \\
\hline Consumption 2 & 2 & 540,0 & 540,5 \\
\hline Consumption 3 & 3 & 900,0 & 900,0 \\
\hline Consumption 4 & 11 & 900,0 & 900,0 \\
\hline Consumption 5 & 22 & 720,0 & 720,0 \\
\hline Consumption 6 & 33 & 1440,0 & 1440,0 \\
\hline
\end{tabular}

As a second example of optimization algorithms possible application we consider the determination of the head from a fuel pump to achieve a specified difference between the fuel entering the flow tank ( $2 \mathrm{P}$ and $2 \mathrm{~L}$ ) and flowing from it. Figure 5 shows the design scheme 
of the fuel system. Set the difference in fuel consumption for the right tank is $180 \mathrm{~kg}$ and for the left tank is $18 \mathrm{~kg}$.

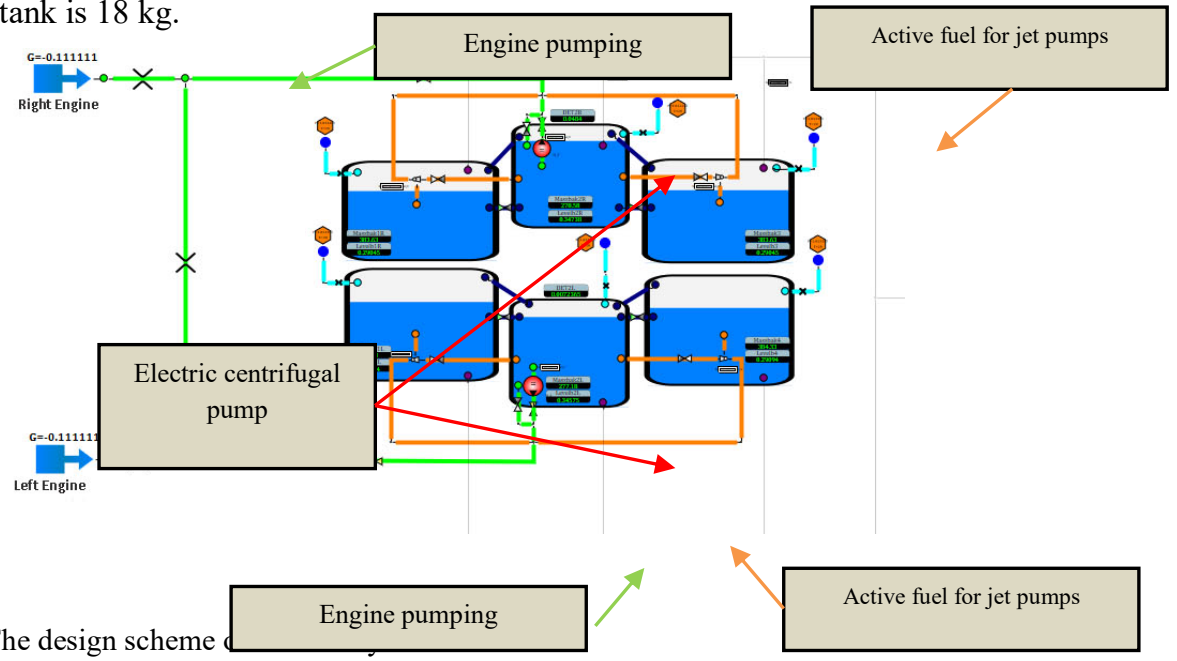

Fig. 5. The design scheme

Like figure 2, the algorithm for selecting the required head from a fuel pump to achieve a given level of the difference in fuel consumption flowing into and flowing out from the flow tank. The calculation also simulates the flow of fuel from the supply tank to other tanks when the overflow level is reached.

Fuel consumption difference values for flow tanks,

$\mathrm{kg} / \mathrm{h}$

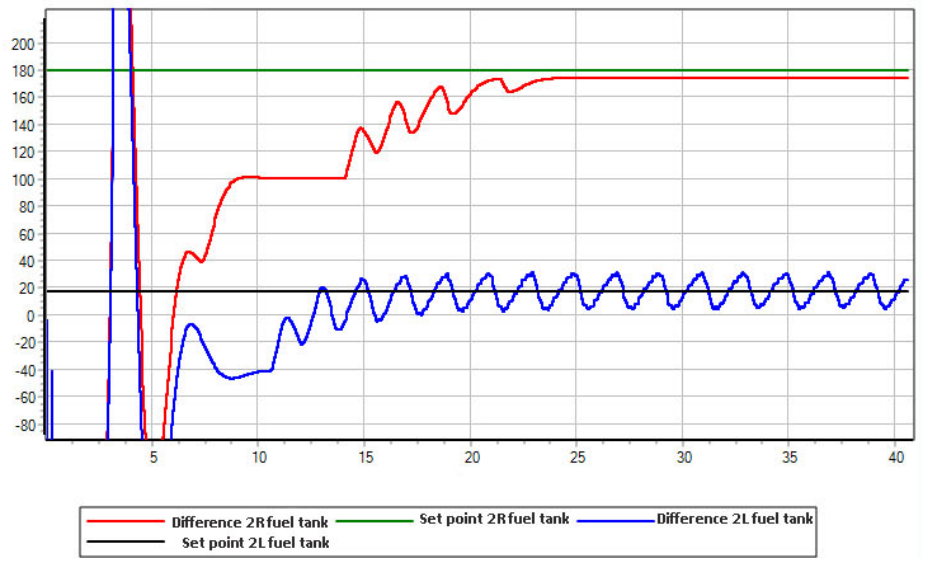

Fig. 6. Fuel consumption difference values for flow tanks 


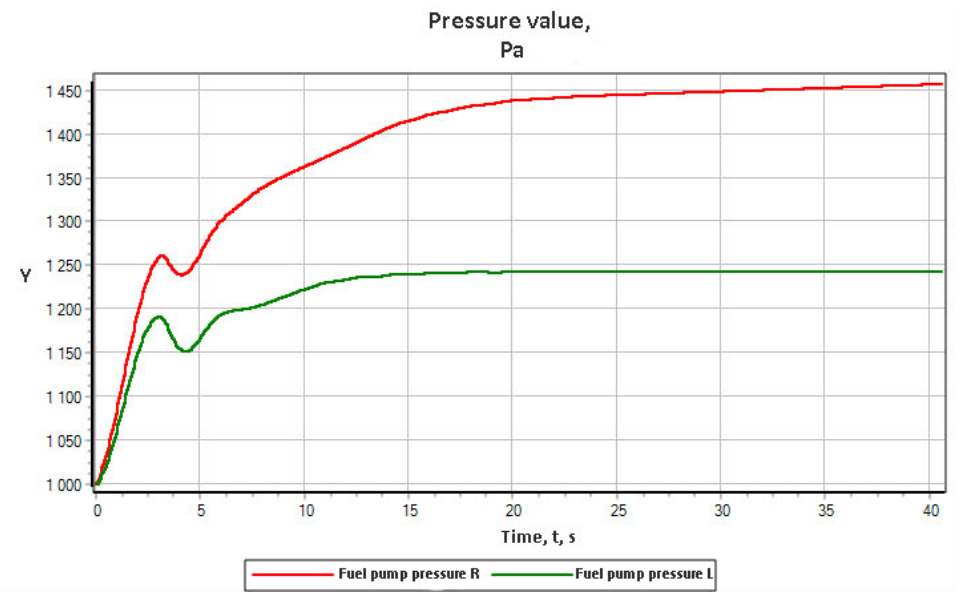

Fig. 7. Pressure values for fuel pumps

Figures 6-7 show the results of the fuel system calculation. As can be seen from figure 6, the set value of the flow difference for the $2 \mathrm{P}$ tank is achieved quite accurately. For the $2 \mathrm{~L}$ tank it is observed an oscillating process, which is primarily associated with a small value of the given fuel consumption difference $(18 \mathrm{~kg})$. [9,10,11,12,13]

\section{Conclusion}

Based on the optimization algorithm examples for determining the design parameters of the fuel system and the parameters of its components, it is obvious that the use of mathematical modeling based on optimization algorithms at the development stage (Gate 2) will allow you to select the parameters of the fuel system quite accurately.

It should be considered that in parallel with the development of the project the mathematical model and its complexity is also developing. One of the development paths of this tool is the integration of one-dimensional models of systems with three-dimensional mathematical models (such as fuel tanks, pipeline sections, etc.) to study physical phenomena such as cavitation or evaluation of uninterrupted fuel supply at all stages of the flight.

Considering the non-stationary modes modeling possibilities of fuel system operation, failure situations, it is obvious that the functionality of the developed dynamic mathematical model in the software complex SimInTech is enough to perform tasks not only at the conceptual design stage, but also at the subsequent stages of the life cycle of fuel system.

\section{References}

1. Innovation process. Model Stage-Gate Idea-to-launch. Stage-gate system Standard. http://stage-gate.com

2. Matveenko A. M., Mechanical equipment systems of aircraft. Moscow, Mashinostroenie Press, 558 p., 2005.

3. E. Ya. Sokolov, I. M. Zinger, Jet Apparatus. - 3rd ed., Moscow.: Energoatomizdat, 352 $\mathrm{p},(1989)$

4. Attekov A.V. Optimization methods / A.V. Attekov, S.V. Galkin, V.S. Zarubin. Publishing House of Moscow State Technical University named after N.E. Bauman, 440p, 2003.

5. Vasilyev, F. P. Numerical methods for solving extremal problems. Science Press, 552p, (1988) 
6. Izmailov, A. F., Solodov M. V., Numerical methods of optimization, Fizmatlit Press, $304 \mathrm{p},(2005)$

7. Study of crash-resistant helicopter fuel systems, final report, aviation research administration Washington, D.C., 20591, (February 2002)

8. N. F. Dubovkin, V. G. Malanicheva, Yu. P. Massur, E. P. Fedorov Physico-chemical and operational properties of jet fuels. Reference, M. Chemistry, 240 p., (1985)

9. SimInTech Manual: http://simintech.ru.

10. N. B. Vargaftik Handbook of thermophysical properties of gases and liquids. 2nd edition., Moscow.: Science, 721 p., (1972)

11. S. Bretschneider. The properties of gases and liquids. Engineering methods of calculation. Translation from Polish; edited by P. G. Romankov. - Leningrad: Chemistry, Leningrad branch, 536 p., (1966)

12. Kartashov B. A., Shabaev E. A., Kozlov O. S., Shchekaturov A. M. Dynamic Modeling Environment of Technical Systems SimInTech. Moscow, DMK Press, 424 p., (2017)

13. Idelchik I. E., Handbook of Hydraulic Resistance. Ed. M. O. Steinberg, 3rd ed., Moscow; Engineering Press, 672 p., (1992)

14. Application of the Simintech software package for mathematical modeling of various onboard aircraft systems (Smagin D.I., Saveliev R.S., Satin A.A.) Abstracts of the XXIII INTERNATIONAL SCIENTIFIC CONFERENCE "SYSTEM ANALYSIS, MANAGEMENT AND NAVIGATION"

15. Methods for the Design of Modern On-Board Systems of Advanced Aircraft (Smagin D., Saveliev R.) Abstracts of the conference «The 10th International Conference on Mechanical and Aerospace Engineering»

16. Formation of the concept of promising aircraft using alternative fuels and energy sources (Smagin D.I., Pritulkin A.A.) Abstracts of the conference "GAGARIN READINGS - 2018"

17. Mathematical modeling of the aircraft fuel system (Yaloso A.V., Kozelkov A.S., Strelets D.Yu., Kornev A.V.) Flight. All-Russian Technical J.

18. Application of the Simintech software package for mathematical modeling of various onboard aircraft systems (Smagin D.I., Starostin K.I., Saveliev RS, Satin A.A.) J. "COMPUTATIONAL NANOTECHNOLOGY"

19. Providing new quality aircraft. Design, manufacture and testing of soft fuel tanks by accidental fuel system of helicopters (Pugachev Yu.N., Makovetsky M.B., Smagin D.I.) "Quality and life" J.

20. Method of creating a dynamic mathematical model of a neutral gas system for a promising aircraft in the SIMINTECH software package (Smagin D.I., Starostin K.I., Savelev R.S., Satin A.A.) J. "COMPUTATIONAL NANOTECHNOLOGY”

21. The concept of creating a complex mathematical model of an aircraft, including the main onboard systems (Smagin D.I., Starostin K.I., Saveliev R.S., Satin A.A.) "Quality and Life" J.

22. Solving the problems of optimizing the design of fuel system units based on additive technologies (Zyazeva T.Yu., Smagin D.I.) "Quality and Life" J.

23. Application of optimization of specified parameters of a crash-resistant fuel system (ASTS) of a prospective helicopter (Smagin D.I., Starostin K.I., Saveliev R.S., Satin A.A.) 